\title{
Taming Chaos by Linear Regulation with Bound Estimation
}

\author{
Jiqiang Wang ${ }^{1}$ and Weijian Chen ${ }^{2}$ \\ ${ }^{1}$ Jiangsu Province Key Laboratory of Aerospace Power Systems, College of Energy \& Power Engineering, \\ Nanjing University of Aeronautics \& Astronautics, 29 Yudao Street, Nanjing 210016, China \\ ${ }^{2}$ College of Aerospace Engineering, Nanjing University of Aeronautics \& Astronautics, 29 Yudao Street, Nanjing 210016, China
}

Correspondence should be addressed to Jiqiang Wang; jiqiang.wang@nuaa.edu.cn

Received 9 October 2014; Revised 18 February 2015; Accepted 16 March 2015

Academic Editor: Her-Terng Yau

Copyright (C) $2015 \mathrm{~J}$. Wang and W. Chen. This is an open access article distributed under the Creative Commons Attribution License, which permits unrestricted use, distribution, and reproduction in any medium, provided the original work is properly cited.

Chaos control has become an important area of research and consequently many approaches have been proposed to control chaos. This paper proposes a linear regulation method. Different from the existing approaches is that it can provide region of attraction while estimating the bounding behaviour of the norm of the states. The proposed method also possesses design flexibility and can be easily used to cater for special requirement such that control signal should be generated via single input, single state, static feedback and so forth. The applications to the Tigan system, the Genesio chaotic system, the novel chaotic system, and the Lorenz chaotic system justify the above claims.

\section{Introduction}

Chaotic phenomena have been gradually recognized as one of the inherent properties of nonlinear dynamical systems since the work of Poincaré, Lorenz, Mandelbrot, and so forth. Indeed, it is an astounding fact that chaos theory has been applied in practically all the scientific disciplines. The widespread recognition of chaos has henceforth sparked extensive research interests in its control. Chaos control has received much attention since the well-known OGY method [1] and the Pyragas method [2]. Indeed the number of publications devoted to chaos control is huge; for example, around 700 references are compiled by Fradkov to the papers published in peer reviewed journals only in 1997-2000 [3]. As a consequence, chaos control has become an important research domain in the theory of nonlinear dynamical systems (e.g., see monographs [4-8]).

Many techniques have been devised for controlling chaos via, for example, nonlinear and robust control, sliding mode control, adaptive control, partial control, control by weak signals, and finite time control ([9-16] and the references therein). In these approaches, the unstable periodic orbits are determined and a control signal is then generated which will stabilize the chaotic system to an equilibrium, locally or globally. In this paper, yet another method is proposed to stabilize a chaotic system. This approach possesses the following two appealing features:

(1) control signal is linear with constant gain matrix. In specific, $u=K x$, where $K$ is constant;

(2) for a particular design $u=K x$, the bounding behaviour of $\|x(t)\|$ is explicitly determined.

Feature (1) means control through static state feedback; therefore, this can simplify the implementation compared with adaptive approaches; feature (2), however, is even desirable as it implies that the bounding evolution of $\|x(t)\|$ can be estimated a priori. Therefore, it is expected that both the domain of attraction and the rate of convergence of $\|x(t)\|$ can be tuned explicitly. These results form the contributions of the paper. The roadmap is as follows: Section 2 formulates the problem to be addressed and states the main results; Section 3 provides several numerical examples to validate the results. And finally Section 4 concludes the paper.

\section{Linear Regulation of Chaos}

Consider a chaotic system $\dot{x}=f(x)$. After a possibly coordinate transformation, it is brought into the following representation:

$$
\dot{x}=A x+G(x),
$$


where $A$ is a constant matrix; $G(x)$ is a locally integrable nonlinear function that is not necessarily Lipchitz. Many control design methods can be utilized to regulate the chaotic system. Here one seeks a particularly simple, linear state feedback control law:

$$
u=K x
$$

where $K$ is a constant matrix. By assuming that all system states are measurable, the problem is to find a constant gain $K$, such that the chaotic system

$$
\dot{x}=A x+G(x)+K x
$$

together with initial condition $x_{0}$ is stabilized and the bounding behaviour of $\|x(t)\|$ is determined. Before presenting the main result, the following assumption is made.

Assumption 1. For nonlinear function $G(x)$, there exists an integer $q \geq 1$, such that

$$
\|G(x(t))\| \leq \gamma\|x(t)\|^{q} \quad(\gamma \text { is a positive constant }) .
$$

This assumption essentially says that $G(x)$ is bounded and Lebesgue measurable, henceforth not necessarily Lipschitzian. The main result can now be stated as follows.

Theorem 2 (chaos control by linear regulation with bound estimation). With the above assumption, the system (1) controlled by the state feedback control $u=K x$ is exponentially stable if $K$ is designed such that all the eigenvalues of matrix $(A+$ $K)$ have a strictly negative real part with the initial condition which satisfies

$$
\left\|x_{0}\right\|^{q-1}<\frac{|\lambda|}{\gamma M^{q}}
$$

where the constants $M>0$ and $\lambda<0$ are specified by

$$
\left\|e^{(A+K) t}\right\|<M e^{\lambda t}, \quad \forall t \geq 0 .
$$

Furthermore, the state $x(t)$ is explicitly bounded by

$$
\|x(t)\| \leq \frac{M\left\|x_{0}\right\| e^{\lambda t}}{\left(1-\gamma M^{q}\left\|x_{0}\right\|^{q-1} /|\lambda|\right)^{1 /(q-1)}} .
$$

Proof. See the Appendix.

Several observations follow immediately from the above important result:

(1) Firstly, for a particular design $u=K x$, the chaotic system can be exponentially stabilized for any initial condition within $\left\|x_{0}\right\|^{q-1}<|\lambda| / \gamma M^{q}$.

(2) Secondly, a design $u=K x$ determines the constants $M$ and $\lambda$, and this subsequently determines the region of attraction as well as the bounding properties of $\|x(t)\|$ in terms of (7). Henceforth, the evolution of $\|x(t)\|$ and allowable initial conditions can be determined a priori.
(3) Thirdly, if a large region of attraction and a fast rolloff rate of $\|x(t)\|$ are desired, one can choose the static gain $K$ making the real part of $\max (\operatorname{eig}(A+K))$ small enough, and this may lead to high gain control, but a "wise" combination of states feedback can provide much improved performance. This will be illustrated in the next section.

(4) Fourthly, the choice of static gain $K$ can be of importance for implementation; for example, a choice $K=$ $\operatorname{diag}\left(\begin{array}{lll}k_{1} & k_{2} & k_{3}\end{array}\right)$ implies static state feedback, while $K=\operatorname{diag}\left(\begin{array}{lll}k_{1} & 0 & 0\end{array}\right)$ implies single input, single state, static feedback control. This issue will be further discussed in the numerical studies in the next section.

(5) Finally, although not shown here, it can indeed be shown that the proposed design possesses robustness to modelling uncertainty. Hence exponential stability of chaotic systems can be guaranteed with bounded disturbances.

\section{Linear Regulation of Chaos: Robustness Issue}

In the above analysis, linear regulation of chaotic systems is investigated. To have a complete discussion on a design methodology, robustness issue must be considered. In this section, additive uncertainty associated with both state matrix $A$ and nonlinear function $G(x)$ is analyzed.

To proceed, consider the following uncertain system:

$$
\dot{x}=(A+\Delta A) x+G(x)+\Delta G(x)+u,
$$

where $\Delta A$ and $\Delta G(x)$ are the additive uncertainty associated with state matrix and nonlinear function, respectively. To look, in detail, at the effect of uncertainty on design, suppose the following condition holds:

$$
\|G(x)+\Delta A x+\Delta G(x)\| \leq \gamma^{\prime}\|x(t)\|^{q^{\prime}} .
$$

As $\Delta G(x)$ is uncertainty associated with $G(x)$, hence it should have $\|\Delta G(x)\|<\|G(x)\|$. Considering $\|\Delta A x\| \leq\|\Delta A\|\|x\|$ and the assumption (ii) $\|G(x(t))\| \leq \gamma\|x(t)\|^{q}$, it is reasonable to assume $q=q^{\prime}$, while $\gamma \leq \gamma^{\prime}$. Therefore, inequality (9) can now be written as

$$
\|G(x)+\Delta A x+\Delta G(x)\| \leq \gamma^{\prime}\|x(t)\|^{q} .
$$

It is seen immediately from Theorem 2 that, for a stabilizing design $u=K x$, the feasible initial condition now becomes

$$
\left\|x_{0}\right\|^{q-1}<\frac{|\lambda|}{\gamma^{\prime} M^{q}} .
$$

And the state $x(t)$ is now bounded by

$$
\|x(t)\| \leq \frac{M\left\|x_{0}\right\| e^{\lambda t}}{\left(1-\gamma^{\prime} M^{q}\left\|x_{0}\right\|^{q-1} /|\lambda|\right)^{1 /(q-1)}} .
$$

A consideration of $d\|x(t)\| / d \gamma^{\prime}>0$ gives the following result. 
Theorem 3 (robust regulation of chaos). For a stabilizing design $u=K x$, the existence of uncertainty reduces the radius of feasible initial conditions and causes an even conservative estimation of the bounding property for $\|x(t)\|$; in specific, the initial conditions now satisfy $\left\|x_{0}\right\|^{q-1}<|\lambda| / \gamma^{\prime} M^{q}$ and the state $x(t)$ is bounded by

$$
\|x(t)\| \leq \frac{M\left\|x_{0}\right\| e^{\lambda t}}{\left(1-\gamma^{\prime} M^{q}\left\|x_{0}\right\|^{q-1} /|\lambda|\right)^{1 /(q-1)}} .
$$

Proof. A collection of results leading to (8)-(12) gives the desired solution.

Remark 4. The existence of uncertainty leads to conservative estimation and increases of uncertainty bound lead to decreased stability margin. This result is thus compatible with the small gain theorem.

\section{Linear Regulation of Chaos: Numerical Study}

4.1. Tigan System. First consider the newly discovered threedimensional chaotic attractor, the Tigan system [17], given by

$$
\begin{aligned}
& \dot{x}_{1}=a\left(x_{2}-x_{1}\right) \\
& \dot{x}_{2}=(c-a) x_{1}-a x_{1} x_{3} \\
& \dot{x}_{3}=x_{1} x_{2}-b x_{3}
\end{aligned}
$$

with $a=2.1, b=0.6$, and $c=30$. Write (14) into

$$
\dot{x}=\left[\begin{array}{ccc}
-a & a & 0 \\
c-a & 0 & 0 \\
0 & 0 & -b
\end{array}\right] x+G(x),
$$

where $x=\left[\begin{array}{lll}x_{1} & x_{2} & x_{3}\end{array}\right]^{T}$ and $G(x)=\left[\begin{array}{lll}0 & -a x_{1} x_{3} & x_{1} x_{2}\end{array}\right]^{T}$. From $\|G(x(t))\| \leq \gamma\|x(t)\|^{q}$, one can choose $\gamma=1$ and $q=2$. Then notice that $A$ has an unstable eigenvalue at $\lambda_{\text {us }}=6.6761$, and we will now design a stabilizer $u=K x$. To make $(A+K)$ have eigenvalues with strictly negative real parts is easy, but for implementation, one should choose $K$ to be simple; for example, $K=\operatorname{diag}\left(\begin{array}{llll}k_{1} & k_{2} & k_{3}\end{array}\right)=\operatorname{diag}\left(\begin{array}{lll}0 & -40 & 0\end{array}\right)$, that is, $u_{2}=-40 x_{2}$, a single input, single state, static feedback control, which is the simplest of all possible control actions. Now the eigenvalues of the controlled state equation are prescribed at $\lambda_{\mathrm{cl} 1}=-0.6125, \lambda_{\mathrm{cl} 2}=-41.4875, \lambda_{\mathrm{cl} 3}=-0.6$, and then from $\left\|e^{(A+K) t}\right\|<M e^{\lambda t}$ one can designate $M=1$ and $\lambda=$ -0.6 . Finally the range of the initial conditions can be determined from inequality (5), $\left\|x_{0}\right\|^{q-1}<|\lambda| / \gamma M^{q}$; that is, $\left\|x_{0}\right\|<$ 0.6. With initial condition $x_{0}=\left[\begin{array}{lll}-0.3 & 0.4 & 0.3\end{array}\right]^{T}$ (thus $\left.\left\|x_{0}\right\|=0.5831<0.6\right)$, the transient behaviour of $x(t)$ is shown in Figure 1, where it is seen clearly that all states are exponentially stabilized with the simple linear feedback control. The corresponding evolution of $\|x(t)\|$ is shown in Figure 2, and also the theoretical bound computed from (7) is shown, cross validating the theoretical results presented in the main theorem.

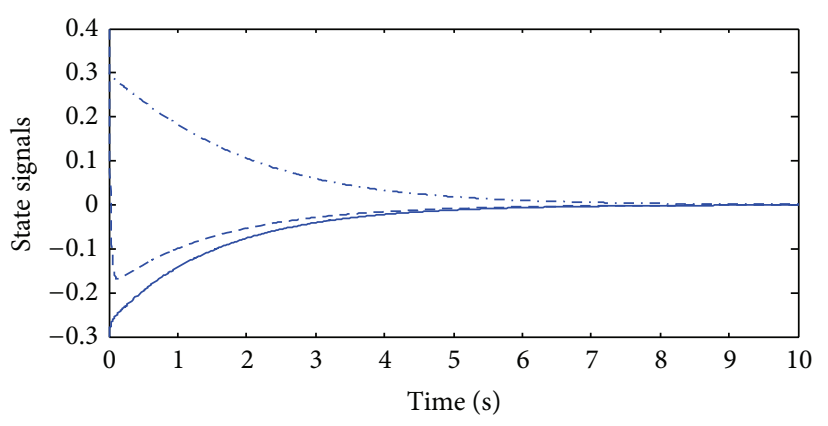

$$
\begin{array}{ll}
- & x_{1} \text { : with control } \\
--- & x_{2} \text { : with control } \\
-.- & x_{3} \text { : with control }
\end{array}
$$

FIGURE 1: Transient response of state signals: closed loop system with $x_{0}=\left[\begin{array}{lll}-0.3 & 0.4 & 0.3\end{array}\right]^{T}$.

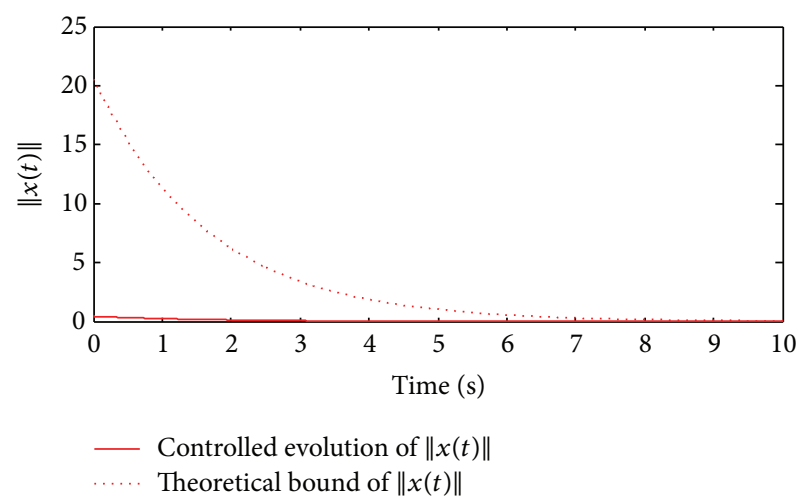

FIGURE 2: The evolution of $\|x(t)\|$ with linear state feedback control $u_{2}=-40 x_{2}$. Also shown is theoretical bound of $\|x(t)\|$ as in (7).

Now two very important observations follow.

(1) Observation I: Enlargement of Domain of Attraction. From Figure 2, it is seen that the estimation of the bound $\|x(t)\|$ is very conservative, and it is wondered whether the region of attraction $\left\|x_{0}\right\|$ is too restricted. This is indeed the case! Take, for example, $x_{0}=\left[\begin{array}{lll}-100 & 100 & 100\end{array}\right]^{T}$ (thus $\left\|x_{0}\right\|=173.2 \gg$ $0.6)$, for the same controller, and the transient behaviour of $x(t)$ is shown in Figure 3. It is seen clearly that even though the initial condition is far outside of the allowable initial conditions, the same controller still exponentially stabilizes the chaotic system.

(2) Observation II: Design Freedom for States Regulation and Detuning. It is also seen that the above control $u_{2}=-40 x_{2}$, although it is the simplest of all possible control actions, results in restricted domain of attraction for initial conditions and "sluggish" regulation of states. Although it has been shown that domain of attraction for initial conditions can be extended far outside, the transient response of $x(t)$ should still be improved. To resolve the problem, it is expected that one should choose even aggressive control action, for example, high gain control. However, as remarked in the 


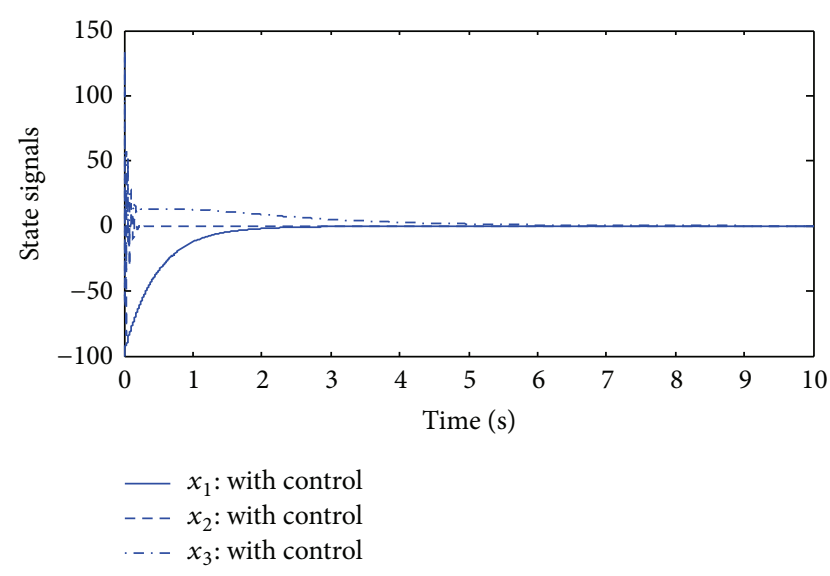

Figure 3: Transient response of state signals: closed loop system with $x_{0}=\left[\begin{array}{lll}-100 & 100 & 100\end{array}\right]^{T}$.

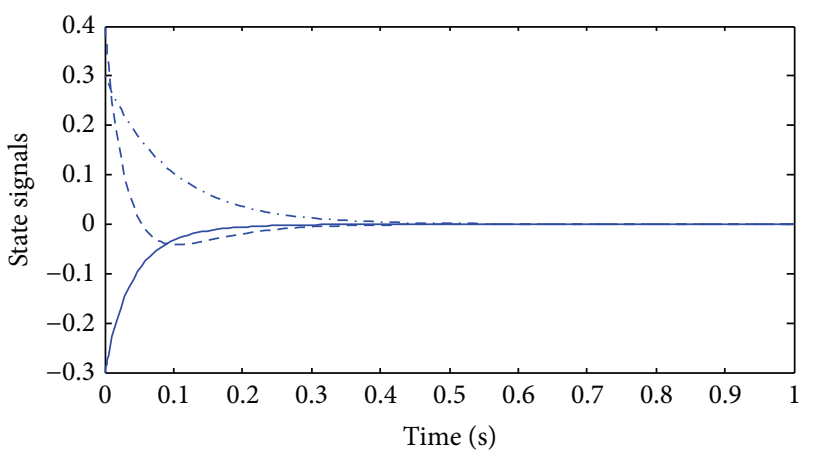

$$
\begin{aligned}
& -x_{1}: \text { with control } \\
& ---x_{2}: \text { with control } \\
& --x_{3}: \text { with control }
\end{aligned}
$$

FIgURE 4: Transient response of state signals with $K=$ $\operatorname{diag}\left(\begin{array}{lll}-20 & -20 & -10\end{array}\right)$.

last section, a wise combination of states feedback can result in improved performance; for example, choose $K=$ $\operatorname{diag}\left(\begin{array}{lll}k_{1} & k_{2} & k_{3}\end{array}\right)=\operatorname{diag}\left(\begin{array}{lll}-20 & -20 & -10\end{array}\right)$; the chaotic system is exponentially stabilized within $0.4 \mathrm{~s}$, compared with $5 \mathrm{~s}$ using $u_{2}=-40 x_{2}$; see Figure 4 .

4.2. Genesio Chaotic System. Now consider the Genesio chaotic system [18] represented by

$$
\begin{aligned}
& \dot{x}_{1}=x_{2} \\
& \dot{x}_{2}=x_{3} \\
& \dot{x}_{1}=a x_{1}+b x_{2}+c x_{3}+x_{1}^{2}
\end{aligned}
$$

with $a=-6, b=-2.92, c=-1.2$. That is, $A=\left[\begin{array}{lll}0 & 1 & 0 \\ 0 & 0 & 1 \\ a & b & c\end{array}\right]$ and $G(x)=\left[\begin{array}{c}0 \\ 0 \\ x_{1}^{2}\end{array}\right]$.

Therefore, from $\|G(x(t))\| \leq \gamma\|x(t)\|^{q}$, we can choose $\gamma=$ 1 and $q=2$. It is also seen that there are unstable eigenvalues at $\lambda_{\mathrm{us}}=0.222 \pm 1.8975 i$, and we will now design a stabilizer
$u=K x$. Here it is remarkable to note that a very simple negative feedback $u_{2}=-x_{2}$ will exponentially stabilize the chaotic system. Again, although the range of initial conditions $\left\|x_{0}\right\|<0.2102(M=1$ and $\lambda=-0.2102)$ appears to be restricted, it can be significantly extended. For example, a simulation with initial condition $x_{0}=\left[\begin{array}{lll}-3 & 4 & 10\end{array}\right]^{T}$ and control action $u_{2}=-x_{2}$ shows exponential stability of the chaotic system; see Figure 5(a). From Figure 5(a), the only concern is the transient performance, and as remarked in the above subsection, a good choice of states feedback can significantly improve the regulation performance. Take, for example, $K=\operatorname{diag}(-10-10-10)$; the situation is shown in Figure 5(b). It is seen that the chaotic system is regulated within $0.4 \mathrm{~s}$, comparing with $20 \mathrm{~s}$ using $u_{2}=-x_{2}$.

4.3. Novel Chaotic System. Now consider the novel chaotic system [19]:

$$
\begin{aligned}
& \dot{x}_{1}=-a x_{1}+x_{2}+b x_{2} x_{3} \\
& \dot{x}_{2}=c x_{2}-x_{1} x_{3}+x_{3} \\
& \dot{x}_{3}=d x_{1} x_{2}-h x_{3}
\end{aligned}
$$

with $a=3, b=2.7, c=4.7, d=3$, and $h=9$. That is, $A=\left[\begin{array}{ccc}-a & 1 & 0 \\ 0 & c & 1 \\ 0 & 0 & -h\end{array}\right]$ and $G(x)=\left[\begin{array}{l}b x_{2} x_{3} \\ -x_{1} x_{3} \\ d x_{1} x_{2}\end{array}\right]$. The system has five equilibrium points that are all unstable saddle focus nodes. But still, it is found that, again, a single input, single state, static feedback $u_{2}=-8 x_{2}$ can achieve exponential stability of the chaotic system, as illustrated in Figure 6(a). The transient performance can be further improved by using two control inputs $u_{1}=-5 x_{1}, u_{2}=-13 x_{2}$, and $u_{3}=0$; that is, $K=$ $\operatorname{diag}\left(\begin{array}{lll}-5 & -13 & 0\end{array}\right)$. See Figure 6(b).

4.4. Lorentz Chaotic System. Let us take yet another example, the unified Lorentz chaotic system [20]:

$$
\begin{aligned}
& \dot{x}_{1}=(25 \alpha+10)\left(-x_{1}+x_{2}\right), \\
& \dot{x}_{2}=(28-35 \alpha) x_{1}+(29 \alpha-1) x_{2}-x_{1} x_{3}, \\
& \dot{x}_{3}=x_{1} x_{2}-\frac{8+\alpha}{3} x_{3},
\end{aligned}
$$

where $\alpha \in[0,1]$. The system reduces to the general Lorenz system for $\alpha \in[0,0.8]$; it is the general Lü system for $\alpha=0.8$ and the general Chen system for $\alpha \in[0.8,1]$. In this study, we take $\alpha=0$ and it is the Lorentz chaotic system with $A=\left[\begin{array}{ccc}-10 & 10 & 0 \\ 28 & -1 & 0 \\ 0 & 0 & -8 / 3\end{array}\right]$ and $G(x)=\left[\begin{array}{c}0 \\ -x_{1} x_{3} \\ x_{1} x_{2}\end{array}\right]$. As again, a single input, single state, static feedback $u_{2}=-40 x_{2}$ can exponentially stabilize the Lorenz chaotic system, as illustrated in Figure 7(a). The transient performance can be further improved by using two control inputs, for example, $u_{1}=-10 x_{1}, u_{2}=-25 x_{2}$, and $u_{3}=0$. But here, to demonstrate the flexibility of the proposed design approach, it is insisted that single input should be used; for example, $u_{1}=-2 x_{1}-$ $50 x_{2}$ while $u_{2}=0$ and $u_{3}=0$. Figure $7(\mathrm{~b})$ illustrates the situation and it is seen that although the transient performance is not improved, exponential stability is achieved through only single input. 


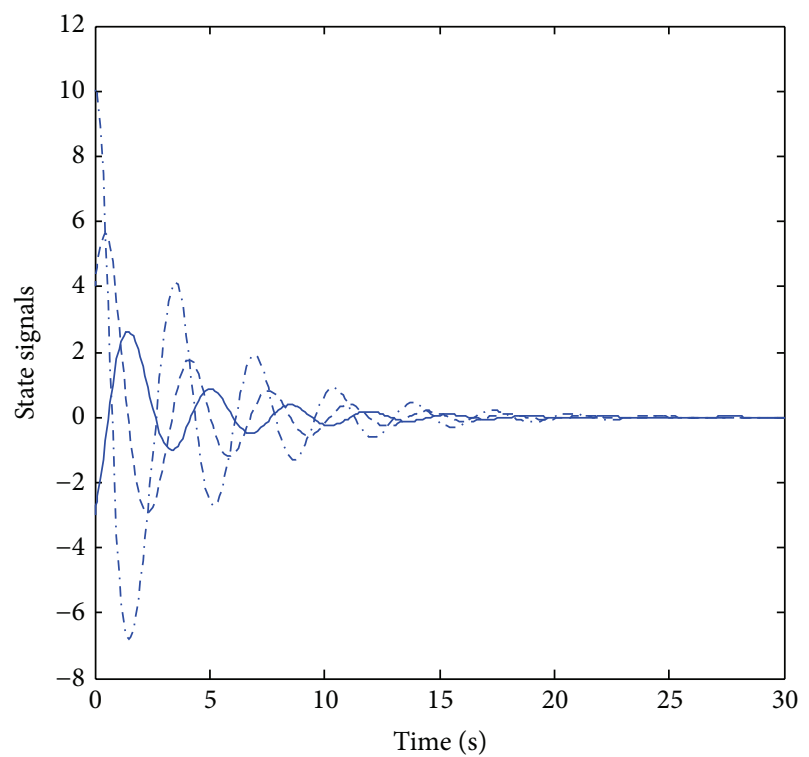

$\begin{array}{ll}- & x_{1} \text { : with control } \\ --- & x_{2} \text { : with control } \\ -.- & x_{3} \text { : with control }\end{array}$

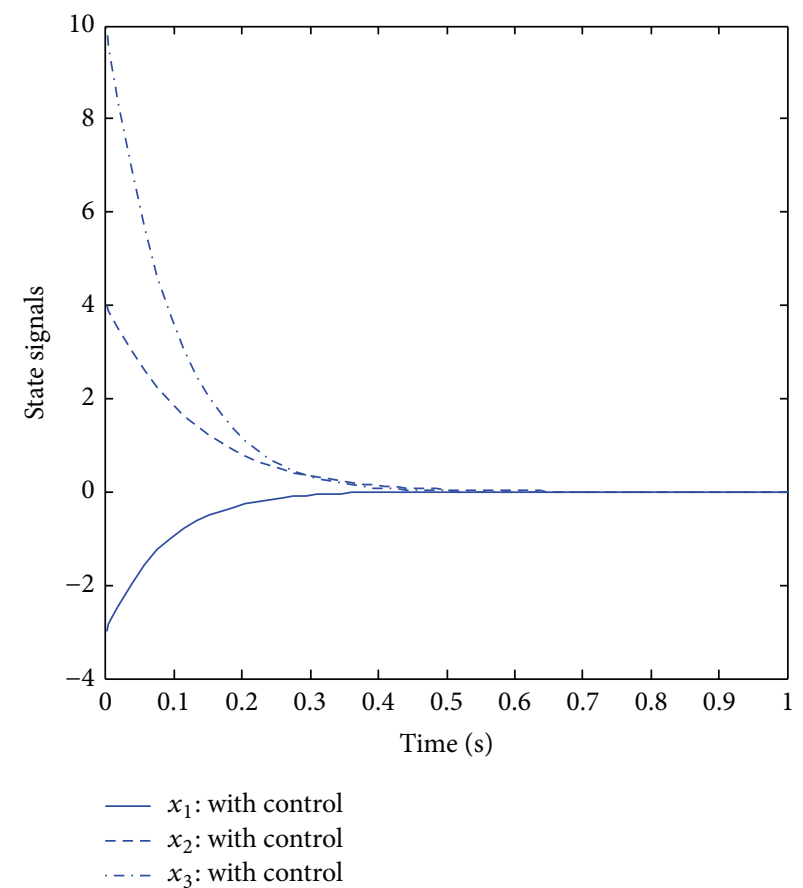

(b)

Figure 5: Transient response of state signals with (a) single input, single state, unit negative feedback $u_{2}=-x_{2}$; (b) static state feedback $u=K x, K=\operatorname{diag}\left(\begin{array}{lll}-10 & -10 & -10\end{array}\right)$.

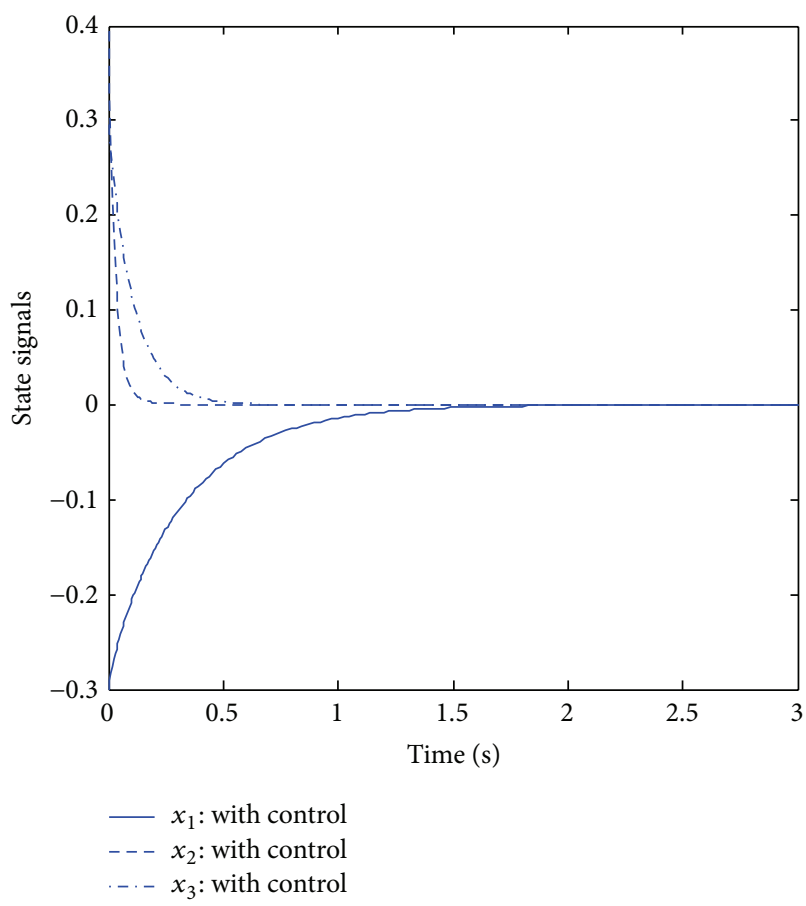

(a)

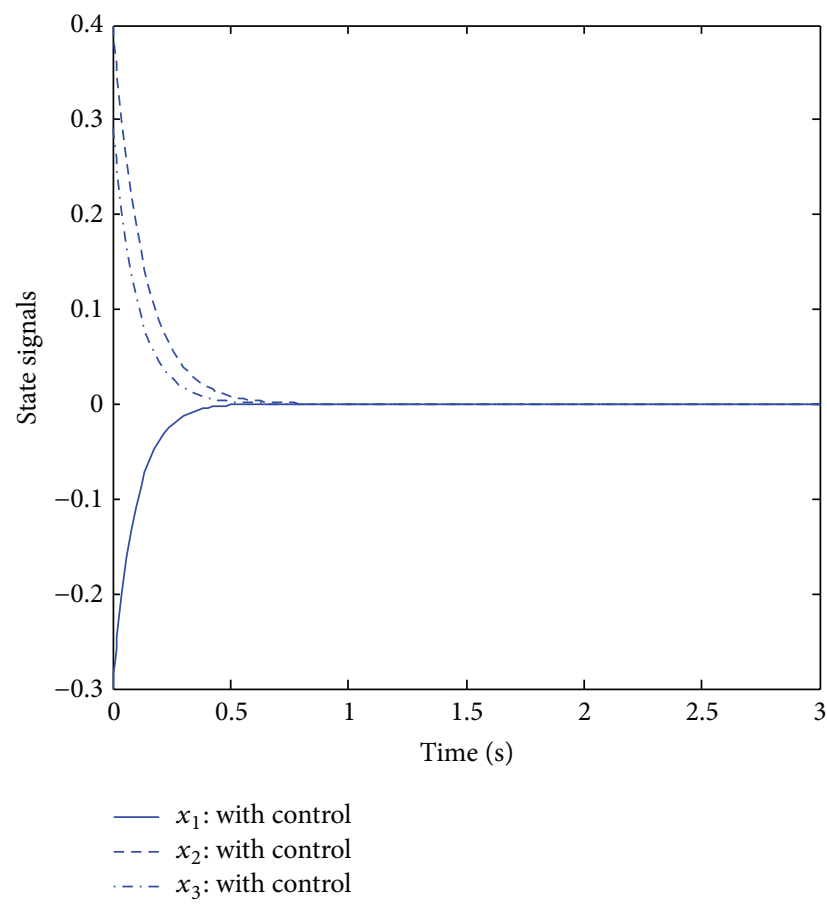

(b)

FIGURE 6: Transient response of state signals with (a) single input, single state, unit negative feedback $u_{2}=-8 x_{2}$; (b) static state feedback $u_{1}=-5 x_{1}, u_{2}=-13 x_{2}$, and $u_{3}=0$. 


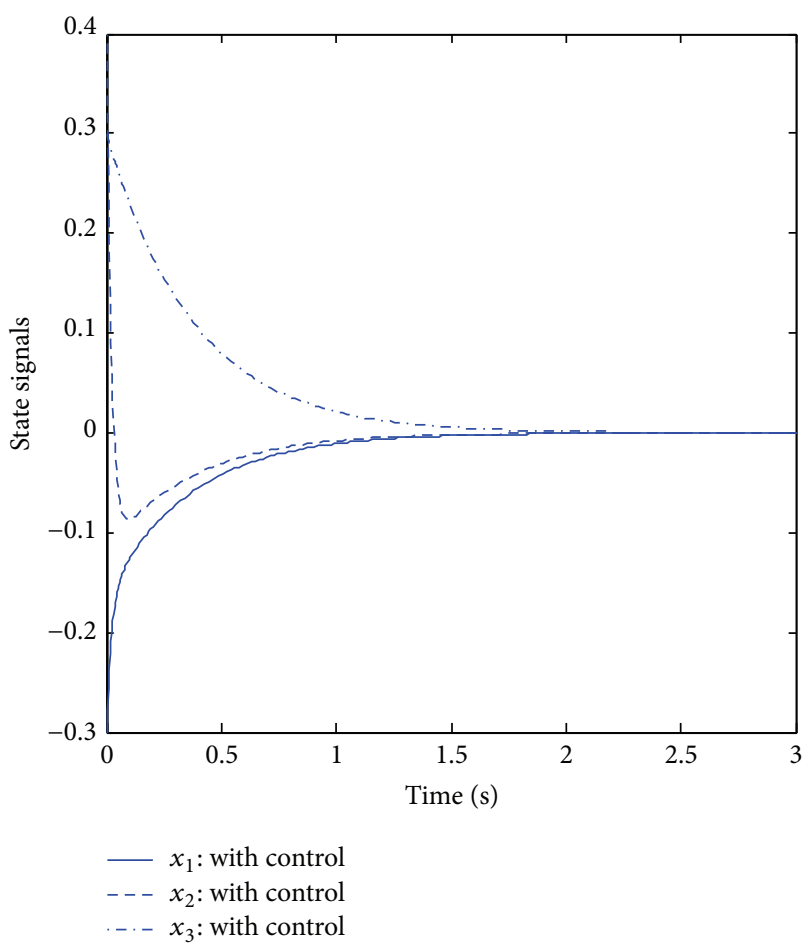

(a)

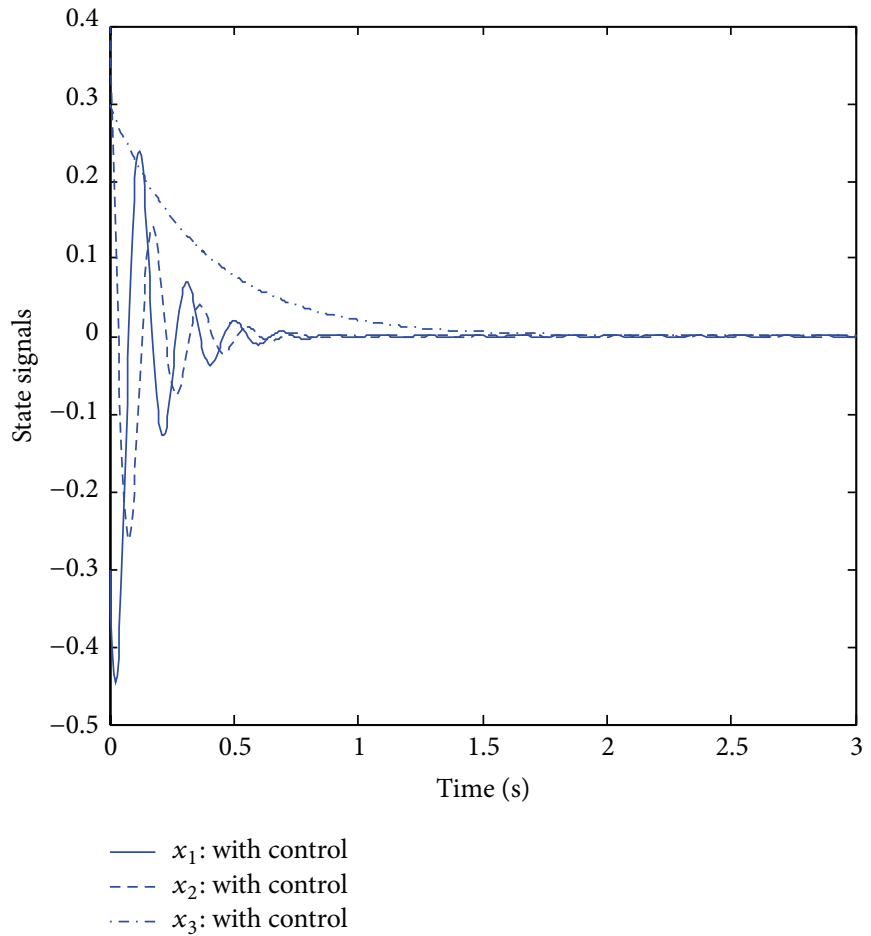

(b)

Figure 7: Transient response of state signals with (a) single input, single state, unit negative feedback $u_{2}=-40 x_{2}$; (b) single input control $u_{1}=-2 x_{1}-50 x_{2}, u_{2}=0$, and $u_{3}=0$.

\section{Conclusion}

Linear regulation of chaos has been introduced in this paper. It has been shown that the proposed design method can provide region of attraction while simultaneously obtaining the bounding behaviour of the norm of the states. The proposed method has also demonstrated its flexibility in design freedom through its application to four types of chaotic systems, namely, the Tigan system, the Genesio chaotic system, the novel chaotic system, and the Lorenz chaotic system. The design flexibility has been clearly revealed by the following facts: (1) all the chaotic systems are exponentially stabilized through single input, single state, static feedback control; (2) transient performance can be improved through appropriate combination of states feedback; and (3) single input, static feedback control is still achievable for exponential stability.

\section{Appendix}

\section{Proof of the Main Theorem}

The proof of the result utilizes the following lemmas.

Lemma A.1 (Gronwall-Bellman lemma [21-23]). Let the following conditions hold:

(i) $f$, $g$, and $k\left(\mathfrak{R}^{+} \mapsto \mathfrak{R}\right)$ are locally integrable, where $g \geq 0, k \geq 0$ and $g \in \ell^{\infty}$;

(ii) $g k$ is locally integrable on $\mathfrak{R}^{+}$.
If $u: \mathfrak{R}^{+} \mapsto \mathfrak{R}$ satisfies

$$
u(t) \leq f(t)+g(t) \int_{0}^{t} k(\tau) u(\tau) d \tau, \quad \forall t \geq 0
$$

then

$$
\begin{aligned}
& u(t) \\
& \leq f(t) \\
& +g(t) \int_{0}^{t} k(\tau) f(\tau) \exp \left(\int_{\tau}^{t} k(s) g(s) d s\right) d \tau, \\
& \forall t \geq 0 .
\end{aligned}
$$

Lemma A.2 (generalized Gronwall-Bellman lemma [24, 25]). Let

(i) $a, b, k \in \Re$ with $0 \leq a<b$ and $k>0$; further define an integer $l>1$;

(ii) $f: \mathfrak{R}^{+} \mapsto \mathfrak{R}^{+}$an integrable function with $\int_{\alpha}^{\beta} f(s) d s>$ $0, \forall \alpha, \beta \in[a, b], 0 \leq \alpha<\beta ;$

(iii) $x:[a, b] \mapsto \mathfrak{R}^{+}$an essentially bounded function such that

$$
x(t) \leq k+\int_{a}^{t} f(s)[x(s)]^{l} d s, \quad \forall t \in[a, b] .
$$


Then if the following inequality holds:

$$
1-(l-1) k^{l-1} \int_{a}^{b} f(s) d s>0,
$$

then one has

$$
x(t) \leq \frac{k}{\left(1-(l-1) k^{l-1} \int_{a}^{t} f(s) d s\right)^{1 /(l-1)}},
$$

$$
\forall t \in[a, b] .
$$

Lemma A.1 is the standard Gronwall-Bellman lemma and it has long been used for the stabilization of nonlinear systems and nonlinear observer design (see [21-23, 26], e.g.). However, for the purpose of the current paper, the original Gronwall-Bellman lemma must be further generalized. One of the most important generalizations to the discussion of this paper, Lemma A.2, is due to Pachpatte [27, 28], El Alami [24], and, more recently, N'Doye et al. [25]. This result will be used in the following for the proof of the main result below.

Now consider the dynamical system (1); with condition (i) in Lemma A.1 the differential equation can be integrated to be

$$
x(t)=e^{(A+K) t} x_{0}+\int_{0}^{t} e^{(A+K)(t-s)} G(x) d s .
$$

Hence

$$
\|x(t)\| \leq M e^{\lambda t}\left\|x_{0}\right\|+M e^{\lambda t} \int_{0}^{t} \gamma e^{-\lambda s}\|x\|^{q} d s
$$

or

$$
\begin{aligned}
\|x(t)\| e^{-\lambda t} \leq & M\left\|x_{0}\right\| \\
& +\gamma M \int_{0}^{t} e^{(q-1) \lambda s}\left(\|x\| e^{-\lambda s}\right)^{q} d s .
\end{aligned}
$$

Here it is time to invoke Lemma A.2, where it can be easily checked that the conditions for the lemma to hold are fulfilled. Hence, provided that

$$
1-(q-1)\left(M\left\|x_{0}\right\|\right)^{q-1} \int_{0}^{t} \gamma M e^{(q-1) \lambda s} d s>0
$$

the behaviour of $\|x(t)\|$ is bounded by

$$
\begin{aligned}
& \|x(t)\| e^{-\lambda t} \\
& \leq \frac{M\left\|x_{0}\right\|}{\left(1-(q-1)\left(M\left\|x_{0}\right\|\right)^{q-1} \int_{0}^{t} \gamma M e^{(q-1) \lambda s} d s\right)^{1 /(q-1)}} .
\end{aligned}
$$

Inequality (A.9) can be further reduced to

$$
1-\frac{\gamma M^{q}\left\|x_{0}\right\|^{q-1}}{|\lambda|}\left[1-e^{(q-1) \lambda t}\right]>0
$$

while (A.10) leads to

$$
\begin{aligned}
& \|x(t)\| \\
& \quad \leq \frac{M\left\|x_{0}\right\| e^{\lambda t}}{\left(1-\left(\gamma M^{q}\left\|x_{0}\right\|^{q-1} /|\lambda|\right)\left[1-e^{(q-1) \lambda t}\right]\right)^{1 /(q-1)}} .
\end{aligned}
$$

A consideration of the inequality $1-e^{(q-1) \lambda t}<1$ gives the desired results.

\section{Conflict of Interests}

The authors declare that there is no conflict of interests regarding the publication of this paper.

\section{Acknowledgments}

This work is supported by the Natural Science Foundation of Jiangsu Province (no. BK20140829) and Jiangsu Postdoctoral Science Foundation (no. 1401017B).

\section{References}

[1] E. Ott, C. Grebogi, and J. A. Yorke, "Controlling chaos," Physical Review Letters, vol. 64, no. 11, pp. 1196-1199, 1990.

[2] K. Pyragas, "Continuous control of chaos by self-controlling feedback," Physics Letters A, vol. 170, no. 6, pp. 421-428, 1992.

[3] A. L. Fradkov, Chaos Control Bibliography (1997-2000), Russian Systems and Control Archive (RUSYCON), 2001.

[4] G. Chen and X. Dong, From Chaos to Order: Methodologies, Perspectives and Applications, vol. 24 of World Scientific Series on Nonlinear Science, World Scientific, Singapore, 1998.

[5] G. Chen, Ed., Controlling Chaos and Bifurcations in Engineering Systems, CRC Press, New York, NY, USA, 1999.

[6] G. Chen and X. Yu, Eds., Chaos Control: Theory and Applications, Springer, Berlin, Germany, 2004.

[7] J. M. González-Miranda, Synchronization and Control of Chaos: An Introduction for Scientists and Engineers, Imperial College Press, London, UK, 2004.

[8] E. Schöll and H. G. Schuster, Eds., Handbook of Chaos Control, Wiley-VCH, Weinheim, Germany, 2007.

[9] D.-Y. Chen, Y.-X. Liu, X.-Y. Ma, and R.-F. Zhang, "Control of a class of fractional-order chaotic systems via sliding mode," Nonlinear Dynamics, vol. 67, no. 1, pp. 893-901, 2012.

[10] R. Zhang and S. Yang, "Stabilization of fractional-order chaotic system via a single state adaptive-feedback controller," Nonlinear Dynamics, vol. 68, no. 1-2, pp. 45-51, 2012.

[11] M. P. Aghababa and H. Aghababa, "Synchronization of nonlinear chaotic electromechanical gyrostat systems with uncertainties," Nonlinear Dynamics, vol. 67, no. 4, pp. 2689-2701, 2012.

[12] C. C. Yang, "Adaptive synchronization of Lü hyperchaotic system with uncertain parameters based on single-input controller," Nonlinear Dynamics, vol. 63, no. 3, pp. 447-454, 2011.

[13] Z. M. Odibat, "Adaptive feedback control and synchronization of non-identical chaotic fractional order systems," Nonlinear Dynamics, vol. 60, no. 4, pp. 479-487, 2010.

[14] S. Bowong, "Adaptive synchronization of chaotic systems with unknown bounded uncertainties via backstepping approach," Nonlinear Dynamics, vol. 49, no. 1-2, pp. 59-70, 2007. 
[15] H. Wang, Z.-Z. Han, Q.-Y. Xie, and W. Zhang, "Finite-time chaos control via nonsingular terminal sliding mode control," Communications in Nonlinear Science \& Numerical Simulation, vol. 14, no. 6, pp. 2728-2733, 2009.

[16] S. Li and Y.-P. Tian, "Finite time synchronization of chaotic systems," Chaos, Solitons \& Fractals, vol. 15, no. 2, pp. 303-310, 2003.

[17] G. Tigan and D. Opris, "Analysis of a 3D chaotic system," Chaos, Solitons and Fractals, vol. 36, no. 5, pp. 1315-1319, 2008.

[18] R. Genesio and A. Tesi, "Harmonic balance methods for the analysis of chaotic dynamics in nonlinear systems," Automatica, vol. 28, no. 3, pp. 531-548, 1992.

[19] S. Dadras and H. R. Momeni, "A novel three-dimensional autonomous chaotic system generating two, three and fourscroll attractors," Physics Letters A, vol. 373, no. 40, pp. 3637$3642,2009$.

[20] J. Lü, G. Chen, and S. Zhang, "Dynamical analysis of a new chaotic attractor," International Journal of Bifurcation and Chaos in Applied Sciences and Engineering, vol. 12, no. 5, pp. 1001-1015, 2002.

[21] B. G. Pachpatte, "A note on Gronwall-Bellman inequality," Journal of Mathematical Analysis and Applications, vol. 44, no. 3, pp. 758-762, 1973.

[22] C. Desoer and M. Viddyasagar, Feedback Systems: Input-Output Properties, Academic Press, New York, NY, USA, 1975.

[23] M. Viddyasagar, Nonlinear Systems Analysis, Prentice Hall, Upper Saddle River, NJ, USA, 2nd edition, 1993.

[24] N. El Alami, "A generalization of Gronwall's lemma," in Proceedings of the International Conference on Differential Equations, Marrakesh, Morocco, 1995.

[25] I. N’Doye, M. Zasadzinski, M. Darouach, N.-E. Radhy, and A. Bouaziz, "Exponential stabilization of a class of nonlinear systems: a generalized Gronwall-Bellman lemma approach," Nonlinear Analysis. Theory, Methods \& Applications, vol. 74, no. 18, pp. 7333-7341, 2011.

[26] K. Shimizu, "Nonlinear state observers by gradient descent method," in Proceedings of the IEEE International Conference on Control Applications, pp. 616-622, Anchorage, Alaska, USA, 2000.

[27] B. G. Pachpatte, "On some generalizations of Bellman's lemma," Journal of Mathematical Analysis and Applications, vol. 51, pp. 141-150, 1975.

[28] B. G. Pachpatte, "On some integral inequalities similar to Bellman-BIHari inequalities," Journal of Mathematical Analysis and Applications, vol. 49, pp. 794-802, 1975. 


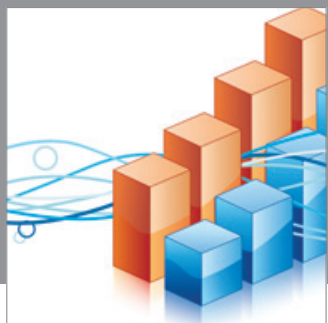

Advances in

Operations Research

mansans

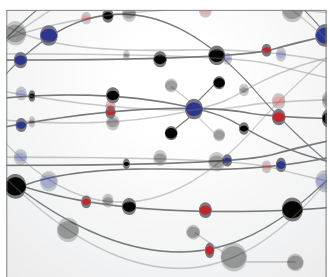

The Scientific World Journal
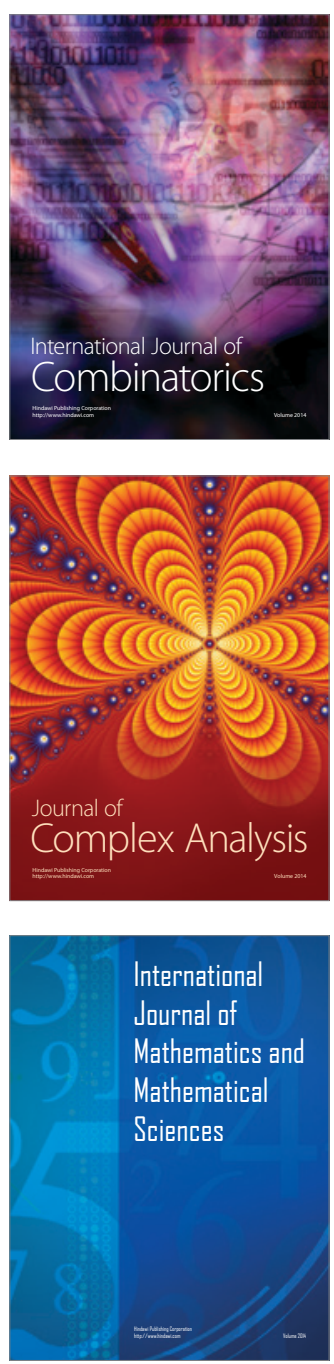
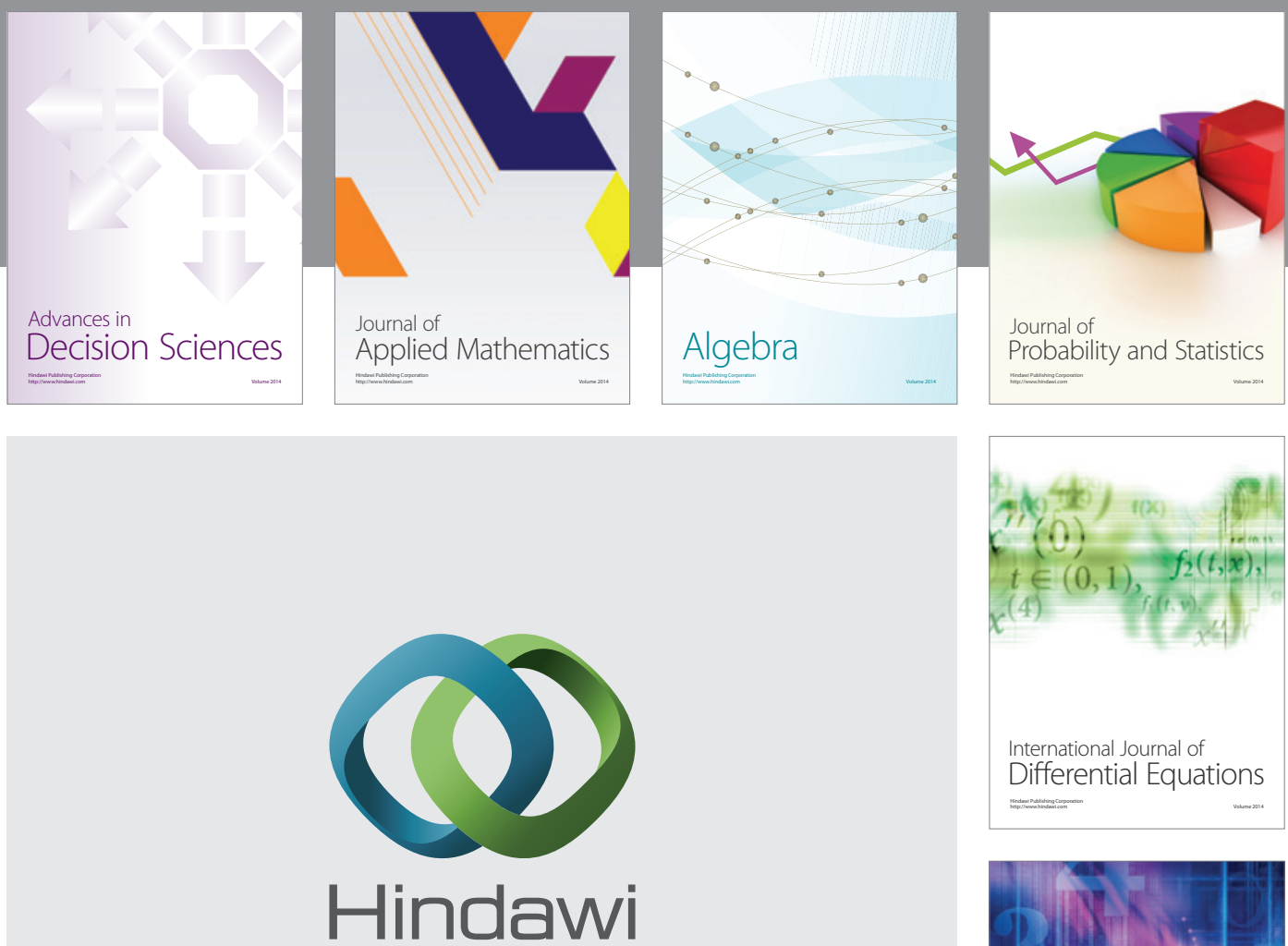

Submit your manuscripts at http://www.hindawi.com
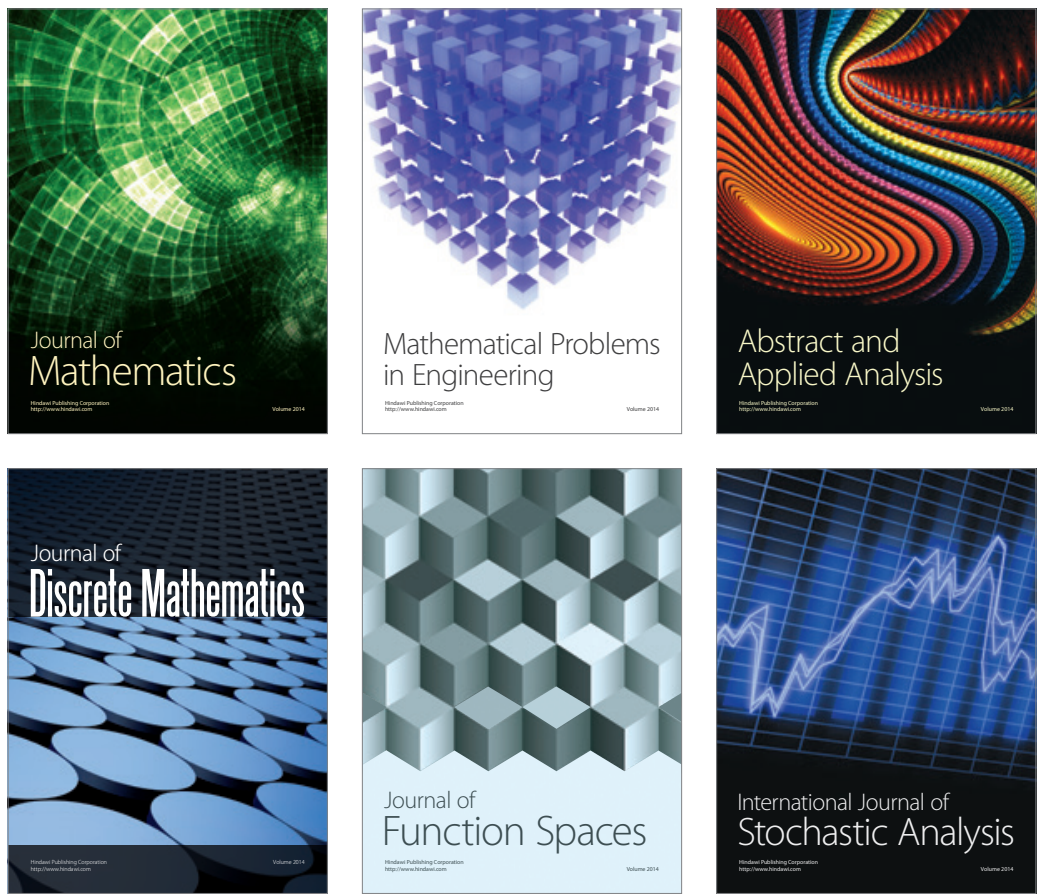

Journal of

Function Spaces

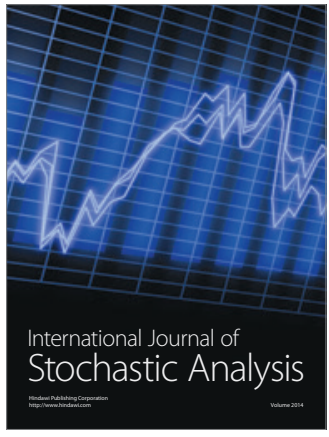

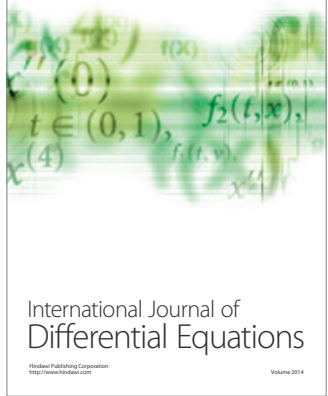
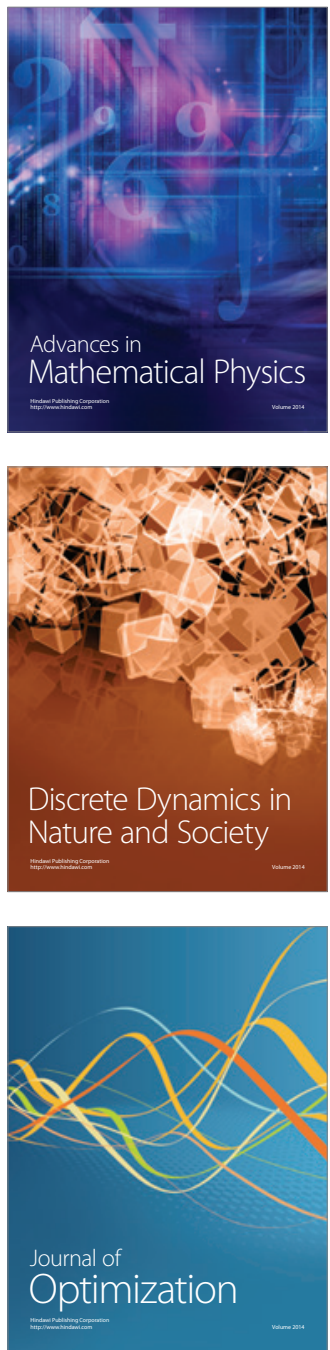